\title{
Der Garten als Mythos
}

\section{Jürgen Strassel, Oldenburg}

\section{Gegenstand und Denkansatz}

Gärten, als Teil unserer alltäglichen Umwelt, haben im letzten Jahrzehnt zunehmend öffentliche Beachtung gefunden. Besonders gilt dies für Ziergärten, und um sie wird es in den folgenden Ausführungen ausschließlich gehen. Nach dem Schönen Wohnen wurden sie in jüngster Zeit in wachsendem Maße Mittel zur Selbstformulierung per Lifestyle sowie Gegenstand gestalterischer Überlegung. Jede Wohnzeitschrift hat inzwischen ihren ausführlichen Gartenteil. Neue Periodika zur Gestaltung und Pflege von Ziergärten, doch auch zur Gartenkunst und ihrer Geschichte sind entstanden. In den Regalen des Buchhandels hat sich die Anzahl von Büchern zum Thema Garten in den letzten beiden Jahrzehnten vervielfacht. Der Kulturtourismus vergrößerte sich in jüngster Zeit um die Abteilung Gartenreisen. Ziergärten als Teil des privaten Lebensraumes sind nach langen Nachkriegsjahrzehnten geringer Beachtung zu einem kulturellen Artikulationsbereich mit steigendem Statuswert geworden.

Die Geographie hat sich wenig um diese Bestandteile von Lebensraum und Kulturlandschaft gekümmert. Obwohl private Gärten einen großen Teil der Siedlungsfläche einnehmen, von eigenwilliger Physiognomie und lebenspraktisch bedeutsame Umwelten sind, gibt es in der deutschsprachigen Geographie bislang nur Ansätze einer Gartenforschung (HARD 1985, 1988, 1998 (in spezifischem Bezug), Strassel 1991, 1992, 1993, Ewald 1993/94, 1999, Paczia 1998). Eine Diskussion um die wissenschaftliche Erklärung der räumlichen Ausprägung und kulturellen Bedeutung von Ziergärten existiert derzeit $\mathrm{m}$. W. in der geographischen Forschung nicht. Meine These angesichts dieses Mangels ist, daß der Geographie eine hinreichend entwickelte Theorie zur Erklärung solcher ästhetischen Phänomene fehlt und dies wiederum in der paradigmatischen Situation des Faches seine Ursachen hat. Die Geographie weist zwar beachtliche ökosystemische, historisch-genetische, sozialstrukturelle, funktionalistische, verhaltens- und handlungstheoretische Erklärungsbedürfnisse und -formen auf, doch zu erklären, warum Menschen einen Teil des ihnen verfügbaren hausnahen Freiraums ohne ökonomischen Sinn und oftmals ohne sozialen Zwang ästhetisch in einer bestimmten Weise gestalten, ist ihr eine eher ungewohnte Zumutung. Eine sozialgeographische Forschung, die sich als Kulturwissenschaft versteht, sollte sich diesem Mangel stellen.
Nun will ich keineswegs den Anschein erwecken, daß ich eine solche Theorie mitsamt tragfähiger paradigmatischer Fundierung liefern könnte. Aber ich kann einige Überlegungen anbieten, die am Beispiel eines kleinen Ausschnitts aus der derzeit bestehenden Gartenkultur auf theoretische Zusammenhänge verweisen, die in Richtung auf eine allgemeinere Reflexion des Phänomens entwickelbar sind. Ich entnehme die Bausteine hierzu strukturalistischen und poststrukturalistischen Forschungszusammenhängen, suche sie semiotisch auszuwerten und so geographisch nutzbar zu machen.

Als erstes möchte ich das Objekt meiner Überlegungen deutlich machen, indem ich - stellvertretend für viele - einen Garten vorstelle, der als einer der einflußreichsten für die derzeit in Europa und in den USA vorherrschende populäre Mode in der Gestaltung von Ziergärten gilt: den Garten von Sissinghurst Castle in Kent, Südostengland, den die Schriftstellerin Vita Sackville-West und ihr Mann Harold Nicolson in den dreißiger Jahren des 20. Jahrhunderts anlegten und der heute zu den meistbesuchten Gärten der Welt zählt. Dann werde ich skizzenhaft eine strukturalistische Mythentheorie referieren und aufzeigen, inwiefern sie mir zur Interpretation von Gärten des gewählten Typus geeignet erscheint. Ein semiotisches Verständnis dieser Interpretation vermag den ahistorischen Charakter dieser Mythentheorie aufzuheben, so daß schließlich in einem dritten Schritt die Bedingungen einer diskurstheoretischen Bestimmung des Forschungsgegenstandes deutlich werden können.

\section{Ein Beispiel: der Garten von Sissinghurst Castle}

Der Garten von Sissinghurst Castle wurde auf dem ca. 4 ha großen Gelände zwischen den Ruinen des in seinen ältesten Teilen elisabethanischen Schlosses angelegt (von den zahlreichen Publikationen über diesen Garten und seine Schöpfer seien hier nur einige genannt: ScotT-James 1987, BRown 1987, 1990, GLENDINNING 1990). Die Reste des alten Baukörpers Mauern und andere Bauteile in unterschiedlichstem Erhaltungszustand - dienten zur Trennung der einzelnen Teilräume, aus denen der Garten besteht. Jeder der Teilräume hat ein anderes Thema, das seine Gestaltung bestimmt. Eine alte Gebäudemauer mit Fensterund Türöffnungen trennt einen Raum mit gemischten Rabatten und Rasen von dem Rosengarten mit seiner streng geometrischen Binnengliederung aus Eibenund Buchshecken. Eine andere Restmauer, ergänzt 


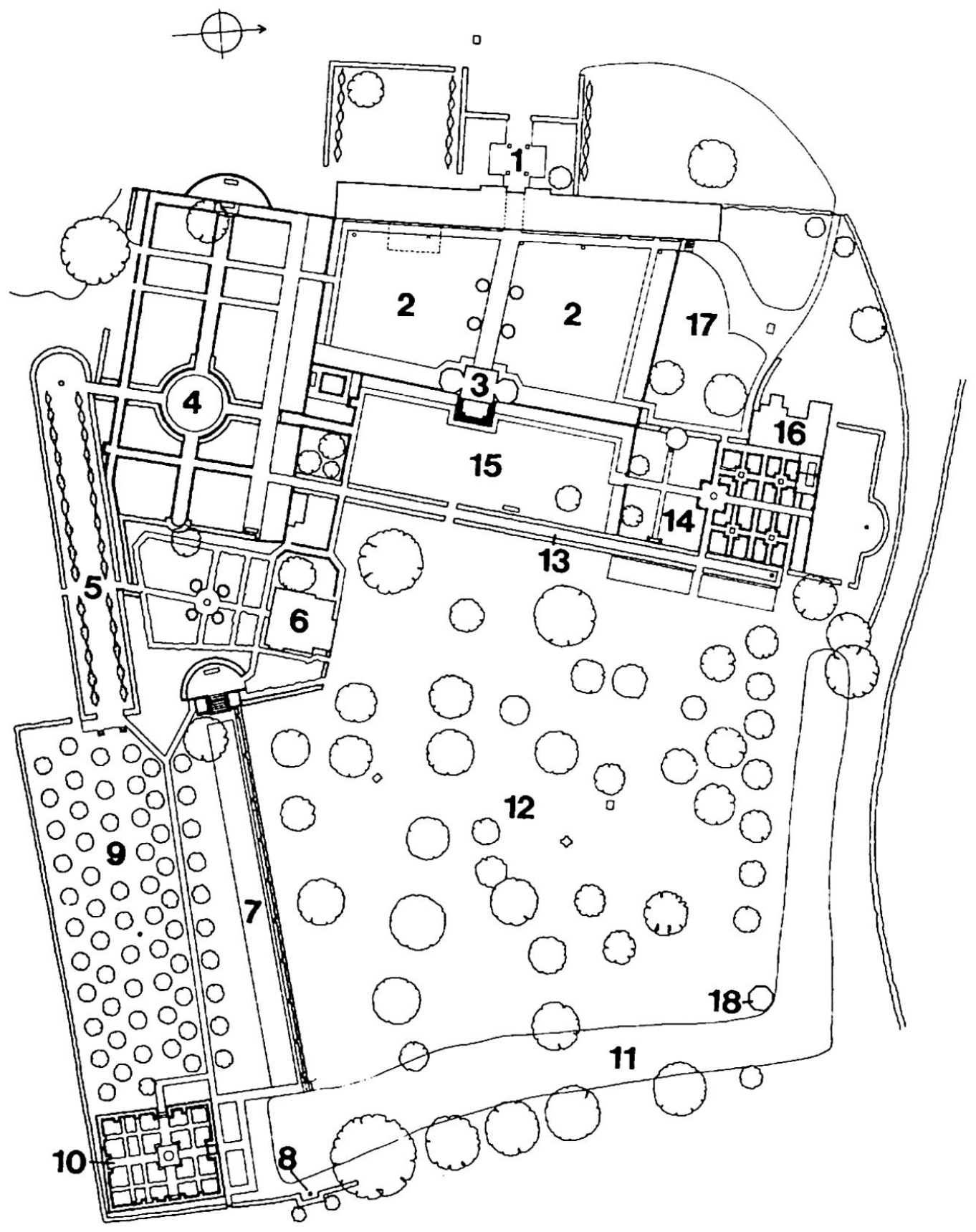

1 Eingang

2 Hofrasen

3 Vitas Turm

4 Rosengarten

5 Lindengang

6 South Cottage und sein Blumengarten

7 Weg zum Wassergraben mit Azaleen

8 Dionysos-Statue

9 Nuttery
10 Kräutergarten

11 Alter Wassergraben

12 Obstgarten

13 Eibengang

14 Weisser Garten

15 Turmrasen

16 Priest's House

17 Delos

18 Gazebo 


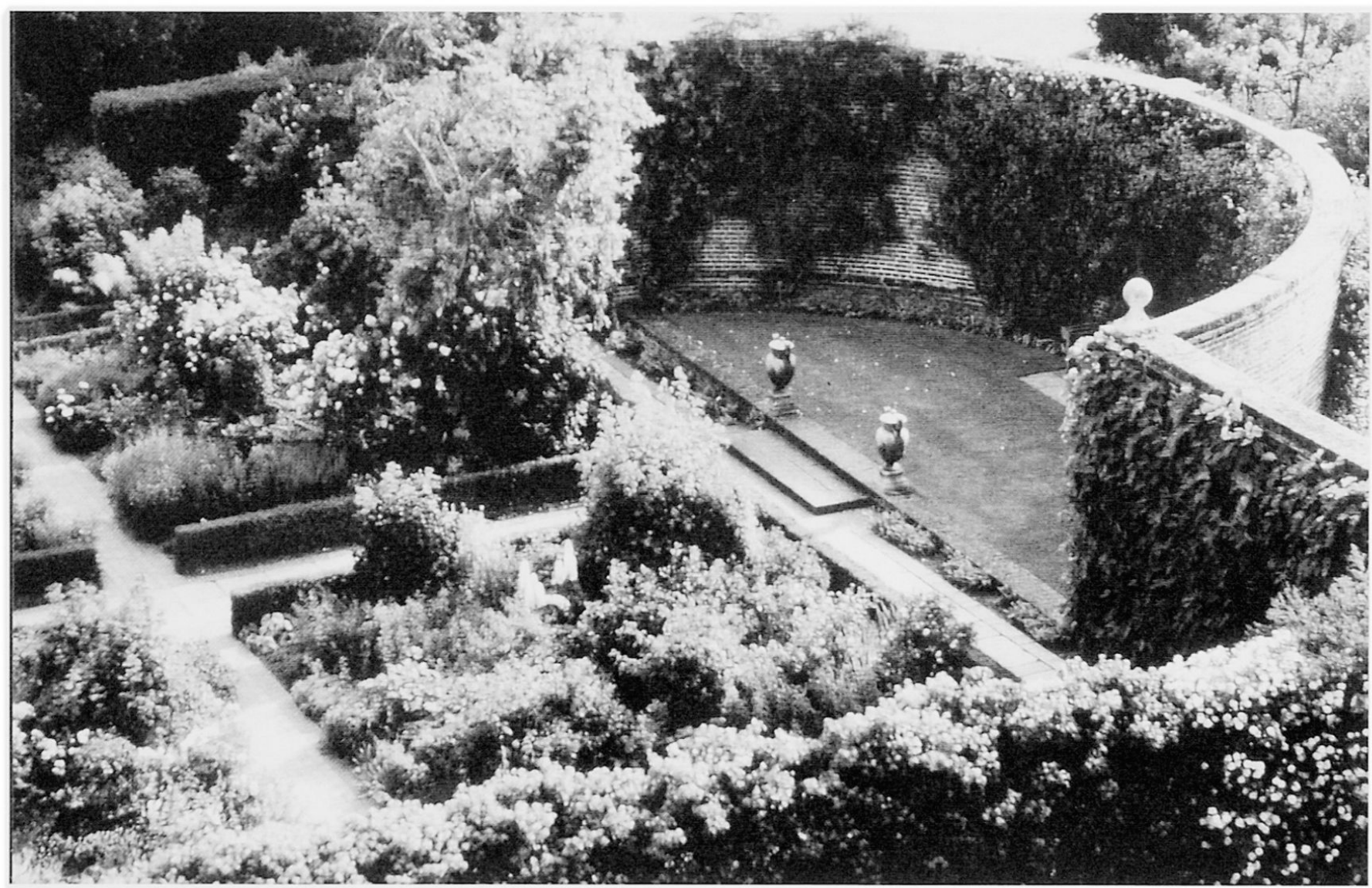

Abb. 2: Sissinghurst Castle: Rosengarten

Photo: J. STRASSEL

Sissinghurst Castle: rose garden

Sissinghurst Castle: jardin des roses

durch hohe, geschnittene Hecken und ein Gebäude, trennt einen Raum mit ausschließlich weißblühenden oder weißblätterigen Pflanzen von einem solchen mit schattenliebenden Stauden- und Buschbeständen, «Delos» genannt, sowie dem Außenraum. Ein dekorativer Kräutergarten und Hecken- und Rasenräume ganz in Grün wechseln mit Gartenteilen mit Rabatten in ausgewählten Blütenfarben: einem Sommergarten in Rot und Gelb, einer Staudenrabatte in blauen und violetten Tönen. Rasengänge, von Ziersträuchersammlungen begrenzt, durchziehen die Anlage. Ein Wassergarten schließt sie gegen das Umland ab. Die Wohnräume der Familie waren in einzelnen Gebäudeteilen über das gesamte Gartengelände verstreut. Der Garten wurde dadurch im Alltag vielmals durchschritten und so intensiv als Teil des Wohnbereichs erlebt.

Der Garten verwirklicht eine Gestaltungsidee, die seit dem Ende des 19. Jahrhunderts - der Ausrichtung englischer Privatgärten auf die Mode historisierender Revivals besonders aus vorgeorgianischer Zeit - den Grundriß größerer Gartenanlagen bei Landhäusern bestimmt: Gärten werden konzipiert als formell-geometrische Anlagen, die das Haus als Architektur und Lebensraum über die Gebäudegrenzen hinaus fortsetzen, so daß die Gartenteile als Zimmer im Freien erlebt werden können. Der Gartengrundriß von Sissinghurst Castle Garden und die räumliche Anordnung der Gestaltungselemente der Gartenanlage zeigen diese Verpflichtung auf einen geometrischen Grundriß deutlich - eine räumliche Ordnung, die im klaren Widerspruch zu den im 18. und 19. Jahrhundert vorherrschenden informellen Anlagen steht. Ihre modische Relevanz war den Zeitgenossen bekannt. Die Wahl dieses Grundrisses muß deshalb als bewußte Gestaltungsentscheidung verstanden werden. Der Einbezug der alten architektonischen Gebäudestruktur der Schloßanlage zur Gliederung des Gartens entsprach diesen Vorstellungen vollkommen (Abb. 1).

Ebenso bewußt ist die Stilisierung der vegetabilen Elemente zu Phänomenen von ganz gegenteiligem Charakter. Die Bepflanzung des Gartens zeigt im Gegensatz zu seiner Architektur eine scheinbar spontan wuchernde Fülle lebendigen Wachstums, die die geo- 


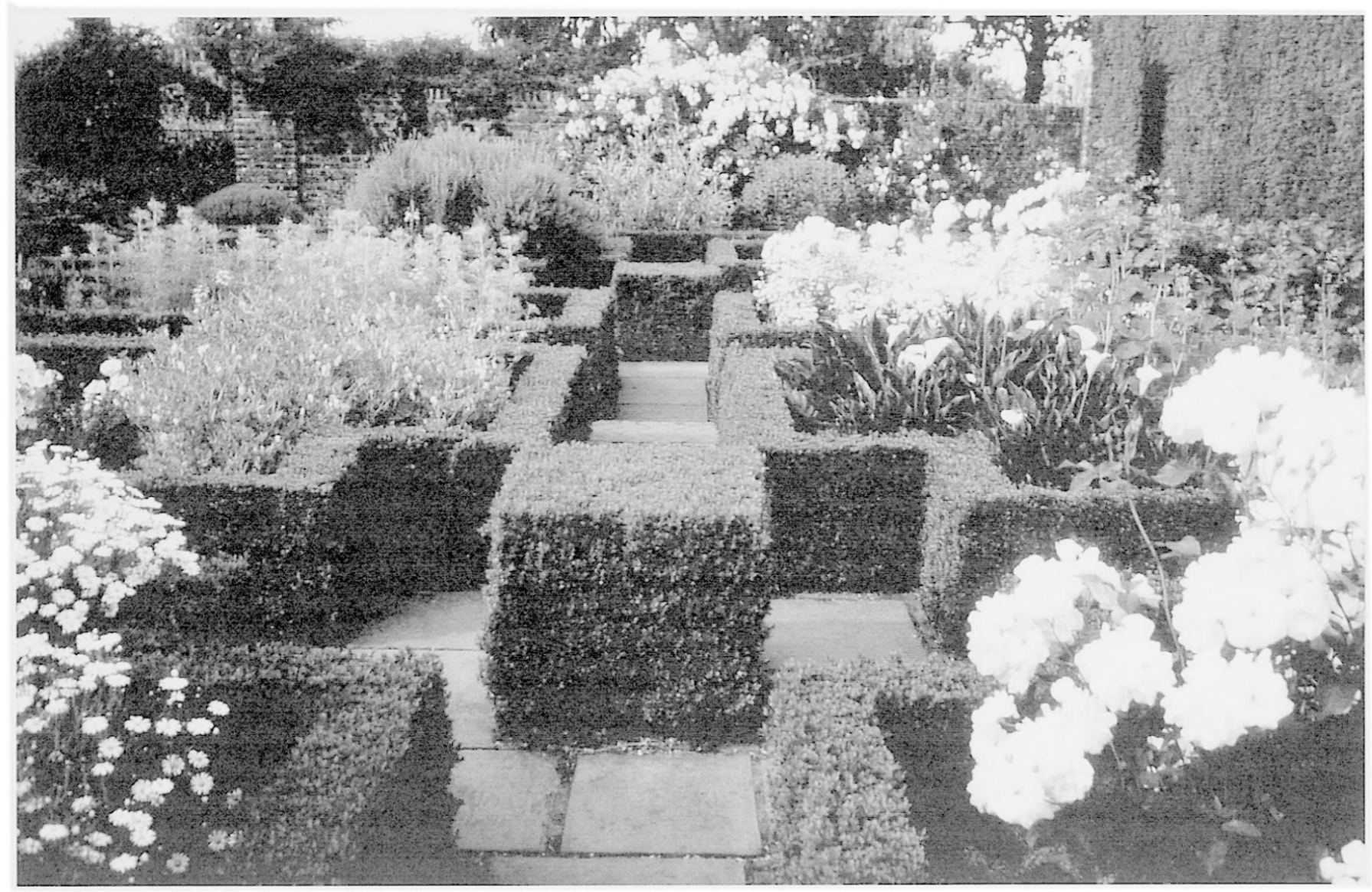

Abb. 3: Sissinghurst Castle: Weisser Garten

Photo: J. Strassel

Sissinghurst Castle: white garden

Sissinghurst Castle: jardin blanc

metrische Strenge von Wegen und Hecken überwindet und die architektonischen Grenzen gleichsam außer Kraft setzt. Der Starrheit strenger gebauter Klarheit und Ordnung wird die überschäumende Wachstumskraft lebendiger Vegetation entgegengesetzt - beide als bewußte und gepflegte Stilmittel, aus deren Gegensätzlichkeit die Gestaltung ihre innere Spannung bezieht. Diese Formenwahl und ihre Deutung als Gegensatz von Ordnung und Fülle, Starrheit und Lebendigkeit und ihre antinomische Wirkung sind für diesen Gartenstil typisch und in der zugehörigen Gartenliteratur vielfach programmatisch festgelegt. Seine Herstellung gilt den Schöpfern solcher Gärten als Bedingung für das Gelingen ihrer Arbeit.

Szenen aus drei Räumen im Garten von Sissinghurst Castle mögen dies verdeutlichen. Der Rosengarten ist einem strengen Muster von Rechtecken und Kreisbögen unterworfen. Die niedrigen geschnittenen Hecken, die die Beetränder begleiten, scheinen oft kaum ihre Aufgabe, die Masse der mannshohen Rosenbüsche und der Stauden zu begrenzen, erfüllen zu können. Erst die massive Gartenmauer bietet ein hinreichendes optisches Gegengewicht zur pflanzlichen Fülle (Abb. 2). Im Weißen Garten akzentuiertsich das gleiche Verhältnis eher umgekehrt: die hohe Heckenrahmung der kleinen Beete und die Dichte des geometrischen Wegenetzes bilden eine Form, die dem Blütenflor, der sie überragt, eher als ein Behältnis zu dienen scheint. Der Bezug ähnelt dem von Vase und Strauß - und hebt die scheinbare Informalität der Pflanzen um so deutlicher hervor (Abb. 3). Im Kräutergarten zeigen besonders die mediterranen Pflanzen zumeist eine geringere Heftigkeit des Wachstums und unauffälligere Blüten. Sie sind deshalb in sorgfältiger Abstimmung von Wuchs- und Blattform, Farbe und Gestalt als ornamentale Ensembles komponiert. Die Wege und die geschnittenen dunklen Eibenhecken der Gartenumrahmung liefern dazu die kontrastierenden, das Muster ergänzenden ruhigen Teile des Bildes. Eine große Schale zentriert es optisch. Es entsteht ein spürbarer Gegensatz zwischen der Vielfalt und dem Abwechslungsreichtum der Kräuter und der beruhigenden Wirkung 


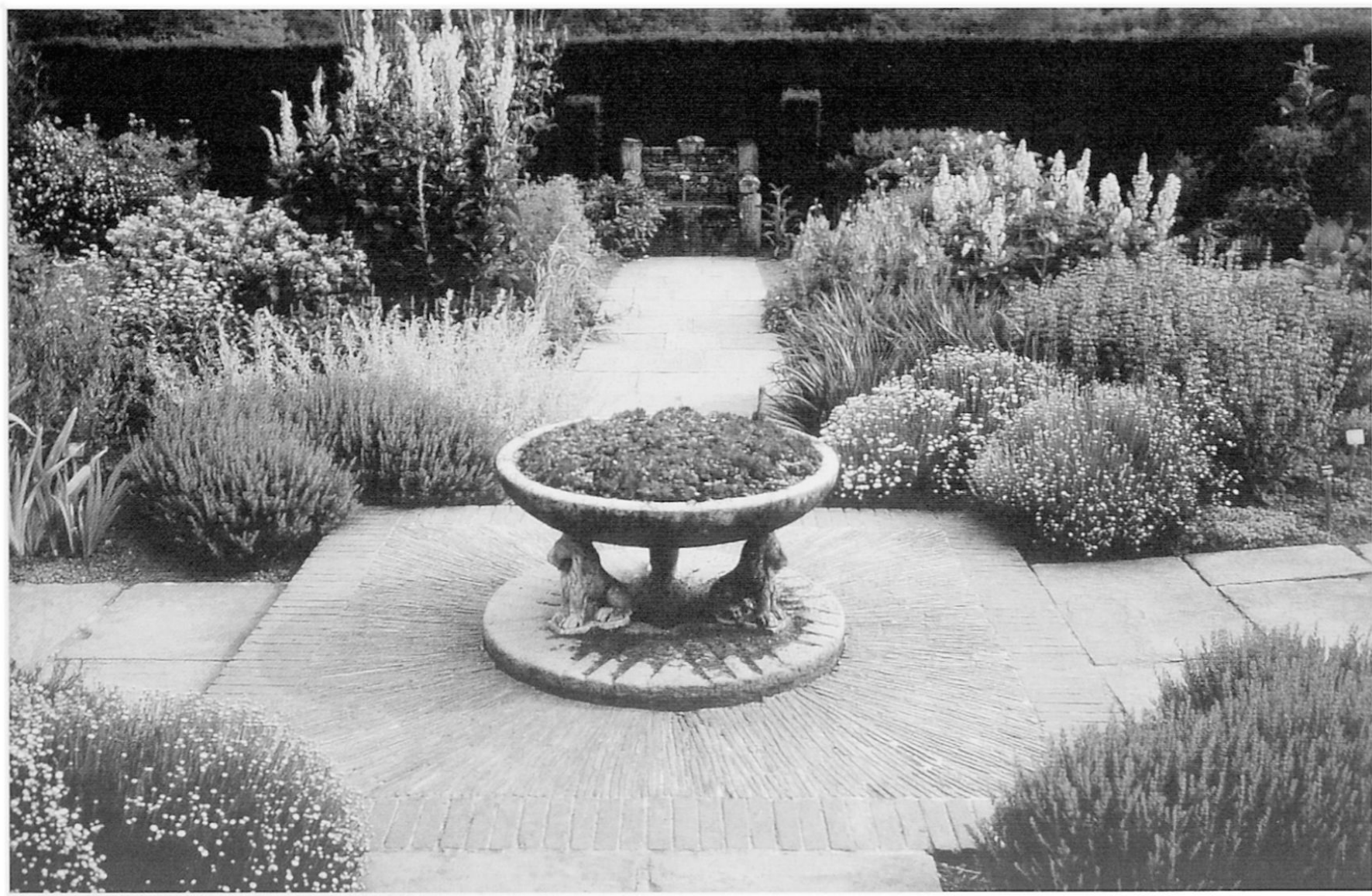

Abb. 4: Sissinghurst Castle: Kräutergarten

Photo: J. STRASSEL

Sissinghurst Castle: herb garden

Sissinghurst Castle: jardin des herbes

der einfachen architektonischen Formen (Abb. 4). Vita SACKVILLE-WEST geht in ihren biographischen wie poetischen und gartentheoretischen Arbeiten immer wieder auf derartige Manifestationen des Gegensatzes von Vegetation und Architektur ein und bindet dabei die beiden Ebenen an die Gegensätzlichkeit einer Reihe individualpsychologischer und gesellschaftlicher Phänomene, vor allem den Gegensatz der Geschlechter, der sich für sie selbst im Garten in der Arbeitsteilung zwischen ihr als der Gärtnerin und ihrem Mann als dem Planer der gebauten Gartenteile ergibt (Sackville-West 1988, Strassel 1991: 117-119). Auch die Oppositionen abstrakter Verhältnisse - etwa von Natur und Gesellschaft oder Sexualität und Moral werden mit den dichotomen Wirkungen von Gartenarchitektur und Gartenbepflanzung parallelisiert. Zugleich weist Vita Sackville-West den Garten als das Reich der Schönheit, des Friedens und der natürlichen Ordnung aus gegen die Wirren und Zwänge der Gesellschaft jenseits ihrer Gartenmauern und die Schrecken der «aus den Fugen geratenen Zeit» in dem inzwischen ausgebrochenen Zweiten Weltkrieg (SACKVILLE-WeST
1946). Der Garten ist ihr der Raum und das Symbol der Hoffnung und der privaten Rettung vor der Welt und dem Leid, das dem Menschen von ihr droht. Er ist, soweit dies möglich ist, das Paradies auf Erden.

\section{Theorie 1: Mythostheorie}

Mit dieser semantischen Zuweisung - der Garten als das Paradies auf Erden - steht Vita SAcKvilleWEST in der Gartenliteratur keineswegs alleine. Das 20. Jahrhundert ist voll von solchen Gleichsetzungen. und das Schrifttum zur gegenwärtigen Gartenmode überstrapaziert diesen Vergleich. Es dürfte fast schwierig sein, einen Text über Gärten im Bereich der Lifestyle- und Infotainmentpublikationen zu finden, der sich nicht der offensichtlichen Eingängigkeit dieser bedeutungsstarken Gleichsetzung bedient und sie anschaulich konkretisiert.

Dieser Sprachgebrauch verführt nun rasch dazu, sich auf der Suche nach einer die Interpretation voran- 
treibenden theoretischen Einpassung des Phänomens «Ziergarten heute» im Reservoir ideologisch wirksamer Denkfiguren und Kollektivsymbole umzuschauen. Der Schritt von der sprachlichen Paradiesmetapher - ob in verbaler oder räumlicher Formulierung zur metasprachlichen Mythostheorie ist dabei naheliegend, zumal eine der anspruchsvollsten Mythostheorien - die des anthropologischen Strukturalismus nach Claude LÉvi-Strauss - mit großem Gewinn den Nachweis gerade oppositioneller Bedeutungsebenen zur Erklärung der ideologischen Wirkung von Mythen betreibt (LÉvi-Strauss 1967: bes. Kap. 11, Gallas 1989). LÉvi-Strauss fragt, ausgehend von seiner Analyse antiker und indianischer Mythen, nach der Struktur des Mythos und seinen tiefenstrukturellen Regeln, um Aufschluß über das mythische Denken als eines universellen anthropologischen Phänomens zu erhalten. Er stellt fest, daß Mythen Vermittlungsversuche zwischen kulturellen Antagonismen darstellen, die die menschliche Grunderfahrung des Bruchs zwischen den Paradigmata «Natur» und «Kultur» zu bewältigen suchen und so die individuelle und soziale Integration der Mitglieder einer kulturellen Gemeinschaft stärken. Mythisierendes Verfahren bei den verbalen Texten, die LÉvi-Strauss untersuchte, ist die Gegenüberstellung binäroppositioneller Bedeutungsträger, besonders von Symbolen, Charakteren und Ideologemen aller Art. Versöhnt werden sie durch ihren Einbezug in eine narrative Konstruktion, die zwischen ihnen vermittelt, ohne sie aufzuheben, beispielsweise, indem sie eine sinnvolle Erzählung mit ihnen formuliert.

Das Paradies der Genesis ist ein Ort solch mythischer Leistungen (Die Bibel1969: Genesis 2 und 3). In ihm sind einige der grundlegenden Gegensätze des menschlichen Lebens nicht vorhanden, obwohl die Bedeutung ihrer Elemente nur im Gegensatzpaar erkennbar wird. Hier gibt es ein Leben ohne Tod, Friede ohne Streit, Nahrung ohne Arbeit, Nacktheit ohne Scham, Geburt ohne Schmerzen. Erst der Sündenfall und seine Bestrafung als narrativ formulierte Kausalsequenz lassen die Begriffspaare als lebenspraktische Widersprüchlichkeiten, die uns allen nur zu bekannt sind, virulent werden. Die Verwirklichung des Paradieses auf Erden heißt, diese Gegensätze - unaufhebbar wie sie im Leben des Menschen sind - zu versöhnen, so daß trotz ihres Bestehens ihr leidvoller Effekt akzeptierbar wird. Der Garten, als Paradies auf Erden gemeint, soll dies leisten - nicht als narrativer Text, sondern als erlebbarer Ort, dessen Gestaltung den Regeln der Mythenbildung entspricht. (Zur Wirkweise architektonischer Codes: Eco 1972). Die gestalterische Ausformulierung der Bereiche "Architektur» und «Vegetation» schafft - wie dies am Beispiel Sissinghurst benannt wurde - die als antagonistisch interpretierbaren Bedeutungsebenen, das ästhetische Programm der Gesamtanlage harmonisiert sie wie- derum. Räumliche Gestaltung und verbale Interpretation wirken in diesem Fall noch zusammen und konkretisieren die Referenzbereiche: im Erleben des gestalteten Gartens soll die Versöhnung des Widerspruchs von symbolisch aufgezeigter gesellschaftlicher Ordnung und individuellem Wachstum, dieser immer wieder benannte Antagonismus in psychologischen wie soziologischen Theorien des Individuums in der bürgerlichen Gesellschaft, als ästhetisches Verhältnis symbolisch erlebbar und akzeptierbar werden.

Die mythenbildende Struktur, die dieser Paradiesdeutung zugrunde liegt, beschränkt sich übrigens keineswegs auf die Mythologie von Gegenwartsgärten des Typus, den Sissinghurst verkörpert. Längst hat sie vom Garten an sich Besitz ergriffen und Gärten ganz unterschiedlicher Gestaltungen und historischer Stile werden nach diesem Deutungsmuster interpretiert. Beim formellen französischen Garten des 17. Jahrhunderts soll es der Gegensatz von Naturbeherrschung und Wildnis sein, ausgedrückt durch die Bodenmuster, die Pflanzenbehandlung und die Figurenprogramme im Garten, die einen Gegensatz zum nicht ästhetisch gestalteten Raum außerhalb des Gartens bilden, in welchem sich der Umgang der Macht mit Natur und Gesellschaft darstellt und aufgrund seiner künstlerischen Herkunft Akzeptanz fordern darf. Im englischen Landschaftsgarten des 18. Jahrhunderts sieht ein Teil des gartenhistorischen Schrifttums das selbstbewußt sich formulierende Individuum des englischen Liberalismus in ein Verhältnis zur Welt, Gesellschaft und Natur tretend - ein Weltentwurf, in dem das gelungene Gesamtkunstwerk die gesellschaftlichen Gegensätze und die neuen Formen der verstärkten Naturausbeutung, die die politischen und ökonomischen Umwälzungen zugleich zeitigten, mit den Mitteln der Natur- und Geschichtsinterpretation zu einem ästhetischen Ganzen kittet. In neuesten Gartengestaltungen schließlich proben Ökologie und Symbolik den anderen Umgang mit innerer und äußerer Natur und ihre private Rettung. Immer gibt die mythenbildende Struktur der Versöhnung realer Antagonismen das Modell für das semiotische System ab, dessen der Garteninterpret sich bedient. Auch wenn es nicht immer die Paradiesvorstellung selbst ist, die dem Ergebnis explizit den Namen gibt, wird die mythische Intention unverkennbar deutlich (BEnZINBERG STEIN 1990, Marcus 1990). Sie setzt zwar bei unzweifelhaft existierenden formalen Gegensätzen in historischen und neueren Gestaltungen an, ihre interpretative Herleitung benutzt jedoch eine semantische Figur, die die zeitgenössischen Quellen nicht oder oft nur mit anderer Bedeutung kennen. Dies gilt sogar für den Paradiesbegriff selbst (vgl. etwa PrEST 1981). Ich folgere: Der Garten als Mythos im aufgezeigten Sinn ist ein Bestandteil der Gegenwartskultur. Er realisiert seine mythische Struktur in den Texten von heute, den ver- 
balen wie den räumlichen Zeichensystemen und findet so eine Bedeutung, die die Gegenwart erzeugt, nicht die historische Form. Der Garten als irdisches Paradies ist die zeitgenössische Antwort auf eine menschliche Sehnsucht von heute. Der platte Spruch, daß «der Mythos lebt», findet hier seine strukturalistische Formulierung.

Der Garten ist als ein System von Aussagen mit der Struktur eines Mythos interpretierbar, formuliert nach den Codes der räumlichen Gestaltung und in ihren verbalen Auslegungen. Wir «lesen» in und von ihm als eine seiner Bedeutungen: dies ist ein Paradies auf Erden. Die räumliche Gestaltung des Gartens als Ort ist seine Zeichensyntax. Nach ihren Regeln bilden die Elemente des Gartens als sein Ausdrucksmaterial bedeutungsvolle Einheiten. Dem Garten die Bedeutung eines Paradieses auf Erden zuzusprechen, macht Sinn, da er zeichenhaft die Inhalte wiedergibt, die wir mit dieser Vorstellung verbinden: er ist ein Ort, in dessen Erleben und Interpretation kulturelle Widersprüche unseres Lebens symbolisch versöhnt und damit im Rahmen dieses Sinnhorizontes als akzeptierbar erscheinen. Wahrgenommen wird dies durch unsere Sinne, verarbeitet durch Emotionen und Intellekt. Das Ergebnis für viele, die verstehen, ist eine tiefe Berührtheit. Und dieser Effekt mag als eine der Erklärungen dafür gesehen werden, daß diese mythische Struktur immer wieder in dieser Weise, als Garten, formuliert und ebenso verstanden wird.

Die strukturalistische Analyse, die diese Einsichten theoretisch faßbar macht, erlaubt die Erarbeitung einer anspruchsvollen empirischen Semantik, die über das hier Dargestellte weit hinausgehen kann. Mein sozialgeographisches Forschungsinteresse zielt jedoch noch in eine andere Richtung: es sucht derart sinnvolle Raum- und Landschaftsgestaltungen auf die gesellschaftlichen Zusammenhänge ihrer Existenz hin zu befragen und räumt dabei vielfach den Akteuren, die an den Prozessen der Erstellung solcher Raumgestaltungen beteiligt sind, besondere theoretische und empirische Beachtung ein. Die strukturalistische Analyse dagegen betrachtet die jeweilige Aktualisierung der Formationsregeln der Sprache als Sprachgeschehen nur als Ausgangsmaterial ihrer Untersuchungen und räumt dem Handlungssubjekt lediglich den Stellenwert des eher zufälligen, nach diesen Regeln Agierenden ein. Das Interesse an der Aufhebung dieses Defizits und die Einführung von Aussagen über gesellschaftliche Zusammenhänge als notwendigen Bestandteilen der Erklärung als Frage an unseren Untersuchungsgegenstand formuliert, hieße nun: Welches sind die gesellschaftlichen Bedingungen, unter denen Mythen zum Gegenstand aktueller Sprech- und räumlicher Gestaltungspraxis und damit raumanalytisch interessant werden? Und welche Rolle spielt dabei das, was die Paradiesvorstellung im Garten faßt, als Bedeutungsgehalt solcher Gestaltungen? Oder kurz formuliert: Warum soll der Garten der Gegenwart ein Paradies sein?

\section{Theorie 2: Diskursbegriff}

Die Suche nach Beantwortung dieser Frage führt von den strukturalistischen weiter zu poststrukturalistischen Forschungsansätzen. Besonders in der Diskurstheorie von Michel Foucault sehe ich hier interessante Ansatzmöglichkeiten (Foucault 1971, 1973, 1974, Frank 1983, 1988), auch wenn - oder gerade weil - sie heute in der theoretischen Diskussion nicht mehr so sehr en vogue ist, sondern eher in den weniger aufsehenerregenden Bereichen der vielfältigen empirischen Anwendungsversuche.

Mein Interesse gilt dabei weniger den epistemologischen Fragen und auch nicht den philosophischen im engeren Sinne, die weitgehend an die Entwicklung der Diskussion in Frankreich gebunden sind, sondern den gesellschaftstheoretischen Implikationen seines Diskursbegriffs und den konkreten Folgerungen, die sich hieraus für das Verständnis des Gartens als Mythos von heute ergeben. Auch will ich mir die Kritik FouCAults und der Diskursanalytiker am Strukturalismus schenken und mich ganz auf unsere Hauptfrage beschränken.

Die Diskurstheorie muß in unserem Zusammenhang in der Lage sein, zwei «Lücken» des strukturalistischen Ansatzes zu schließen. Sie muß zum einen den Gedanken einer universalistisch bestimmten Mythosstruktur mit der Existenz konkreter historischer Antagonismen verbinden. Die Widersprüche, die die Gestaltung eines Gartens mythisch versöhnt, sind, kulturell verankert, diejenigen einer konkreten Zeit und Gesellschaft und sie haben in diesen ihre Ursachen, gewinnen aus ihnen ihren Sinn als mythenbildende. Widersprüchlichkeit alleine wirkt noch nicht mythisierend. Und zum andern muß diese Struktur in ihren jeweiligen Realisierungen als Gestaltung eines Gartens in der Analyse an konkrete Handlungszusammenhänge gebunden sein, sonst gewinnt sie keine sozialwissenschaftliche Relevanz.

Der Diskursbegriff ermöglicht es, beiden Forderungen zu entsprechen. Er bezeichnet dabei allerdings weniger ein Produkt kommunikativer Tätigkeit, sondern umreißt in erster Linie eine Reihe von Bedingungen, unter denen kommunizierbare Inhalte zustande kommen und sich mit weiteren Handlungen verbinden. Fast alle Poststrukturalisten - LaCAN wie Derrida, der späte Roland BARTHES ebenso wie der amerikanische Dekonstruktivismus und Foucault - vollziehen dabei eine eindeutige Dezentrierung des Subjekts: 
Texte werden nicht in erster Linie als Elemente semiotischer Handlungszusammenhänge und damit als Produkte eines Sprechers, Gestalters u.ä. analysiert, sondern als Ereignisse, in denen sich eine symbolische Ordnung, die den unter ihrer Geltung sozialisierten Subjekten das Miteinandersprechen und das Miteinanderhandeln erlaubt, realisiert. Diese ist sicherlich dem kommunizierenden und handelnden Individuum gesellschaftlich vorgegeben. Und weiterhin ist diese Ordnung an die gesellschaftlichen Bedingungen gebunden, unter denen sie Geltung hat, so daß es nicht eine universalistisch begründbare, sondern eine Mehrzahl historisch herleitbarer Ordnungen gibt: die Diskurse. Sprecher und Text oder Gestalter und Garten sind damit nicht mehr Ausgangsvoraussetzungen der Analyse, sondern sind den allgemeinen Bedingungen, die den Aufbau dieser Relation steuern, nachgelagert. Die Idee des Gestalters als kreativ-bildendem Subjekt weicht der einer vorgängigen symbolischen Ordnung sowie der Vorstellung vom Einzelnen als Schnittpunkt differenter Diskurse. Die Analyse richtet sich nicht mehr auf das Bewußtsein der Individuen von einer Ordnung der Dinge. Die diskurstheoretische Analyse konzentriert sich vielmehr auf das Feld von funktionierenden Ähnlichkeiten, Serien, Streuungen von Akten diskursiver Praxis. In sie sind die Subjekte inkorporiert und die Analyse fragt nach den sie generierenden Mechanismen. Die einzelnen Texte und Gestaltungen sind dementsprechend nur die Ausgangspunkte der Analyse, deren Elemente im nächsten Schritt diskursiven Aussageformationen bzw. letztlich historischen Macht-Wissen-Konzeptionen zugeordnet werden müssen (FohrmanN \& Müller 1988: 16).

Der Charakter solch historischer Einbindung läßt sich gut an der Art erkennen, wie Foucault sich in der Bestimmung der kleinsten Einheiten seiner Analysen, den Aussagen, gegen die vorherrschenden sprachtheoretischen Modelle absetzt (ich beziehe mich dabei vornehmlich auf verstreute Bestimmungen in FouCAULT 1973). Aussagen sind ihm weder Sätze noch logische Propositionen noch Sprechakte. Sie sind nicht Elemente eines ideellen Systems, sondern müssen als Handlungen erfolgen, die jedoch nicht in ihren Bindungen an Akteure, Signifikanten, Referenten u.ä. gefaßt werden, sondern im Bezug auf ein «Referential», d.i. eine Menge von Gebieten, in denen solche Handlungen materielle Existenz gewinnen. Erst in diesem Zusammenhang ergibt sich ihre jeweilige Subjektbeziehung und das assoziierte Feld, innerhalb dessen Aussagen nur Gültigkeit gewinnen können. Die Analyse der Aussagen ist deshalb weder eine Suche nach Wahrheit, noch nach Bedeutung, noch nach dem Sinn einmaliger subjektiver Äußerungen, sondern eine Auflösung der Komplexität jeweils relevanter sprachlicher Performanzen, die Isolierung von Termini und die Suche nach Regelmäßigkeiten, denen diese Termini gehorchen in der Absicht, ihre historischen Existenzmodalitäten zu bestimmen. Erst dem analytischen Blick erschließt sich die Struktur, die die Aussageereignisse zusammen mit anderen bilden. Und eben dies gilt für den Diskurs, jene geregelte Formation von Aussagen, die durch anonyme historische Regeln, die die Wirkungsbedingungen der Aussagen definieren, im Rahmen von Machtverhältnissen, Institutionen und Wissenskonfigurationen bestimmt sind.

Die als mythische interpretierbaren Gestaltungen und Texte werden somit zu historischen Ereignissen. Ihre Fähigkeit, zwischen antagonistischen Paradigmen zu vermitteln, erweitert sich dabei in modernen Gesellschaftsformationen auf andere als das von LÉvISTRauss festgestellte Tiefenstrukturkonzept «Natur» vs. "Kultur». Mit Foucault verweisen etwa J. Link (Link 1988) und R. Parr (Parr 1992: 41ff.) auf die Tendenzen zur diskursiven Überbrückung der durch die Formen der Arbeitsteilung in modernen Gesellschaften bewirkten Brüche zwischen hochspezialisierten Wissensbereichen, die eigene Diskurse ausbilden, wie sie gerade durch die Fachwissenschaften institutionalisiert werden. Die Verständigung über die Grenzen der Spezialdiskurse hinweg erfolgt dadurch, daß Diskurselemente mit Integrationsleistung interdiskursive Funktionen wahrnehmen können und so die Verständigungsfähigkeit herstellen. Die sprachtheoretisch orientierte Mythenforschung hat hierfür eine Reihe von Beispielen herausgearbeitet und deren Bedeutung im kulturellen Interdiskurs anschaulich gemacht (Link \& WÜLFING 1984, Wülfing, BRUNS \& PARR 1991, PARR 1992). Charakteren wie Narrationsmustern, Symbolen wie Mythen kommt dabei eine ganz besondere Bedeutung zu, da sie in verschiedenen Diskursen Verwendung finden und damit gesellschaftliche Erfahrungs- und Praxisbereiche miteinander verbinden können. Die Medien wie die Künste liefern gleichermaßen, wenn auch unterschiedliche interdiskursive Formen dieser Art (vgl. etwa Link 1986). Ihre gesellschaftliche Bedeutung scheint mir in der Vermittlung spezialdiskursiver Inhalte in alltäglichen und praxisbezogenen Mitteilungs- und Verständigungssituationen zu liegen.

Gärten als Paradiese gedeutet möchte ich dem Bereich solcher «interferrierenden, koppelnden, integrierenden usw. Querbeziehungen zwischen mehreren Spezialdiskursen» (Link \& Link-HeER 1990: 92) zuordnen. Dies erklärt die kulturellen Synthesen, die sie leisten und macht ihren Ereignischarakter und letztlich ihren Sinn aus. Das Verhältnis von Architektur und Vegetation als Gestaltung des Gegensatzes von Kultur und Natur wird in dieser Symbolisierung zu einer «elementaren Anschauungsform» (LiNk 1988: 286), die gerade in der Widersprüchlichkeit, die sie enthält, ihre Inhalte für interpretierende Alltagsdiskurse verfügbar 
macht. Jeweils Spezialdiskursen der Natur- und Kunstwissenschaften entstammend, reden sie als Mythos vom menschlichen Glück, der Sehnsucht nach ihm und seiner Herstellbarkeit. Wie weit das diskursive Feld reicht, auf dem sich die Paradiesvorstellungen - nicht als theologische Objekte, sondern zur Benennung von Wunschorten menschlicher Glücksfindung (vgl. Börner 1984, Bernheim \& STAVRides 1992) - formulieren, läßt die Verbreitung unsortierter Alltagsfunde vermuten. Ein Stadtbummel in einer Mittelstadt bescherte mir schon in kürzester Zeit die Ankündigung von fünf erreichbaren Paradiesen: einem Einkaufsparadies, einem Badeparadies, einem Bettenparadies, einem Kinderparadies und einem Urlaubsparadies. Die Versprechungen von Freizeitprogrammen und Werbekampagnen stellen sich als solche der Befriedigung leicht käuflich erfüllbarer Sehnsüchte heraus, deren Ausformulierung im 18. Jahrhundert als literarische Utopien begann. Und in den Fernsehprogrammen werden als Paradiese gleichermaßen der Garten wie die Wildnis ausgewiesen (als Beispiel für zahlreiche mögliche Nennungen: «Paradiese auf Zeit», 3-teilige Serie in 3Sat 1997, und «Europas wilde Paradiese», 3-teilige Serie im ZDF Dez.1999).

\section{Fazit}

Stellte die strukturalistische Mythentheorie, ausgehend von Mythen als Manifestationen in Erzählform oder erlebbaren Gestaltungen die Frage nach den tiefenstrukturellen Regeln des mythischen Denkens als einem universellen anthropologischen Phänomen (PARR 1992:18f.), so gilt der Diskursanalyse die «Differenz» zwischen Struktur und Ereignis, zwischen der «linguistischen und semiotischen Regularität einer Aussage und ihrem historischen Auftauchen, das ein historisches Ereignis ist" (KOLKENBROCK-NETZ 1988: 273), als Gegenstand. Gärten als Mythos zu interpretieren, heißt, sich in jedem Fall auf die diesen theoretischen Ansätzen inhärente linguistisch-semiotische Grundposition einzulassen, $\mathrm{da} \beta$ das betrachtete Phänomen immer auf etwas anderes, hinter ihm stehendes, verweist, auf dessen Erfassung und regelbezogenes Verstehen das Interesse des Wissenschaftlers zielt. Die gestalteten Räume werden dabei als Texte gefaßt, in denen die allgemeinen Strukturen des sprachlichen Umgangs mit Wirklichkeit zur Konstituierung jeweils gültiger Sinnbestimmungen reflektierbare Form gewinnen und zu Abkömmlingen und Kreuzungspunkten von Diskursen werden, die in dieser gut vermittellbaren Gestalt die Glücksvision als die imaginäre Rettung am privaten Ort mitformulieren. Sie bieten sich uns an als ebenso wenig erforschte wie fruchtbare Manifestationsbereiche sprachlicher Wirklichkeitskonstitutionen, über deren Analyse sich die Regeln unseres Verstehens und Denkens der Welt erschließen lassen, so, wie wir beim reflektierenden Spielen eines Spiels dessen Regeln erkennen und das Spiel verstehen. Zwar sagt uns dies letztlich nur wenig darüber, wie die Welt ist, um so mehr jedoch kann uns deutlich werden, als was und wie wir sie verstehen.

Unter dem konstruktivistischen Vorbehalt, daß ersteres nur als Funktion von letzterem gelingt, verweist uns der Bezug der beiden in einigen Grundgedanken skizzierten textanalytischen Ansätze auf Ziergärten als Erfahrungsobjekte zumindest auf die Erscheinungsformen des historischen Widerspruchs von Individuum und Gesellschaft. Als Annahme ließe sich formulieren, daß die in den letzten beiden Jahrhunderten der technisch-ökonomischen Entwicklung in den bürgerlichen Gesellschaften zugrundeliegenden Erwartungen, daß sich durch Naturbeherrschung und -aneignung die Versprechungen der Aufklärung hinsichtlich eines allgemeinen gesellschaftlichen Fortschritts zunehmend einlösen ließen, mit den Alltagserfahrungen der Individuen in weiten Bereichen nicht deckten. Die Ideenund Wissenschaftsgeschichten zeigen uns im gleichen Zeitraum eine Fülle ideologischer Entwicklungen, die sich um Erklärung und Kompensation dieses Gegensatzes bemühen: von der Relativierung des Wirklichkeitsbezugs individuellen Erkennens von realistischen hin zu phänomenologischen Auffassungen bis zu immer neuen theoretischen Ausformulierungen und Institutionalisierungen herrschender Fortschrittsprämissen in den Fachwissenschaften. Es bedürfte noch vieler empirischer Bausteine, um von diesen Thesen her eine tragfähige Verbindung zum als Paradies interpretierbaren Garten der Gegenwart als einer interdiskursiven Vermittlungsform zwischen der Hoffnung auf individuelles Wohlergehen und den herrschenden Formen des gesellschaftlichen Umgangs mit Natur zu errichten. Der Mythos jedenfalls bewährt sich wohl schon lange bei der Herstellung solcher Versöhnungen. Und er vereint im Garten zugleich symbolisch repräsentierte Grundannahmen herrschender Spezialdiskurse: in der Gestaltung des Gartens die Überzeugtheit von der heilsamen Notwendigkeit des menschlichen Ordnungswillens gegenüber der Umwelt, in der Lebendigkeit des Wachstums und der Schönheit der Gartenpflanzen den Glauben an die letztendliche Unzerstörbarkeit einer dem Menschen dienenden Natur und in der Hingabe an das ästhetische Entzücken die des Verschwindens der Wirklichkeit unter der Vorherrschaft des Erkennens. Für sich alleine haben diese Aussagen keine Bedeutungen, die im Garten aufzufinden wären oder im Zusammenhang mit ihm Sinn machen würden. Als Deutungen symbolischer Formulierungen und im Aufeinanderbezug als Teile einer Gestaltung dagegen wachsen sie zu Elementen einer Weltsicht von interdiskursiver Bedeutsamkeit zusammen. Die Identifikation - nicht die wissenschaftliche Erklärung - des Gartens als Mythos, die Verwirklichung seiner Struktur 
als semantischem Ereignis ist eine Bedingung ihrer Akzeptanz. Daß dies als Interpretation geschieht, verweist zugleich darauf, daß diese Deutung der Gestaltung des Gartens der Fall sein kann, nicht muß, eine Möglichkeit, keine Zwangsläufigkeit des Verstehens ist. Und weiterhin weckt es das Verlangen, das Spiel des Verstehens weiterzutreiben, um die Vielfalt an sinnlichen Erfahrungen, die der gestaltete Ort anbietet, zum Reichtum des eigenen Welterlebens werden zu lassen.

\section{Literatur}

Benzinberg Stein, A. (1990): Thoughts Occasioned by the Old Testament. - In: Francis, M. \& R.T. Hesters jr., (Hrsg.) (1990): The Meaning of Gardens. - London: 38-45.

Bernheim, P.-A. \& G. Stavrides (1992): Welt der Paradiese - Paradiese der Welt. - Zürich: Artemis und Winkler.

BörNER, K.H. (1984): Auf der Suche nach dem irdischen Paradies: Zur lkonographie der geographischen Utopie. - Frankfurt am Main: Jochen Wörner.

Brown, J. (1987): Vita's other world: a gardening Biography of Vita SackvilleWest. - London: Viking.

Brown, J. (1990): Sissinghurst - A Portrait of a Garden. - London: Weidenfeld and Nicolson.

Die Bibel. Die Heilige Schrift des Alten und des Neuen Bundes (1969). - Freiburg: Herder.

Eco, U. (1972): Einführung in die Semiotik. - München: Wilhelm Fink.

EwALD, U. (1993/94): Gärten. Eine Plage, eine wirtschaftliche Notwendigkeit, ein Born der Freude oder ein Objekt geographischer Forschung? - In: Heidelberger Geographische Gesellschaft - Journal 7/8: 120-131.

Ewald, U. (1999): Gardens and Horticulture in the Americas: An Analysis of Divergent Development between Angloamerica and Latin America. - In: Jahrbuch für Geschichte Lateinamerikas 36: 271-293.

FohrmanN, J. \& H. Müller (1988): Einleitung: Diskurstheorien und Literaturwissenschaft. - In: FOHRMANN, J. \& H. Müller (Hrsg.): Diskurstheorien und Literaturwissenschaft. - Frankfurt am Main: Suhrkamp: 9-22.

Foucault, M. (1971): Die Ordnung der Dinge. - Frankfurt am Main: Suhrkamp.

Foucault, M. (1973): Die Archäologie des Wissens. Frankfurt am Main: Suhrkamp.

Foucault, M. (1974): Die Ordnung des Diskurses. Frankfurt am Main: Fischer.

Frank, M. (1983): Was ist Neostrukturalismus? Frankfurt am Main: Suhrkamp.

Frank, M. (1988): Zum Diskursbegriff bei Foucault. In: Fohrmann, J. \& H. Müller (Hrsg.): Diskurstheorien und Literaturwissenschaft. - Frankfurt am Main: Suhrkamp: 25-44.
Gallas, H. (1989): Der Blick aus der Ferne. Die mythische Ordnung der Welt und der Strukturalismus. - In: Kemper, P. (Hrsg.): Macht des Mythos - Ohnmacht der Vernunft? - Frankfurt am Main: 267-288.

Glendinning, V. (1990):Vita Sackville-West. Eine Biographie. - Frankfurt am Main: Frankfurter Verlagsanstalt.

HARD, G. (1985): Städtischer Rasen, hermeneutisch betrachtet. Ein Kapitel aus der Geschichte der Verleugnung der Stadt durch die Städter. - In: Festschrift Elisabeth Lichtenberger. Klagenfurter Geographische Schriften 6:29-52.

HARD, G. (1988): Die Vegetation städtischer Freiräume. Überlegungen zur Freiraum-, Grün- und Naturschutzplanung in der Stadt. - In: Stadt Osnabrück/Der Oberstadtdirektor (Hrsg.): Perspektiven der Stadtentwicklung. Ökonomie - Ökologie. - Osnabrück: 227-244.

HARD, G. (1998): Ruderalvegetation. Ökologie und Ethnoökologie. Ästhetik und «Schutz». - Kassel: Arbeitsgemeinschaft Freiraum und Vegetation (Notizbuch 49 der Kasseler Schule).

KolKenBrock-NetZ, J. (1988): Diskursanalyse und Narrativik. Voraussetzungen und Konsequenzen einer interdisziplinären Fragestellung. - In: FoHrmanN, J. \& H. MüLlER (Hrsg.): Diskurstheorien und Literaturwissenschaft. - Frankfurt am Main: 261-283.

LÉvi-Strauss, C. (1967): Strukturale Anthropologie I. - Frankfurt am Main: Suhrkamp.

LiNK, J. (1986): Elementare narrative Schemata in der Boulevardpresse. - In: KLOEPFER, R. \& K.-D. MÖLlER (Hrsg.): Narrativität in den Medien.- Münster: MAkS, Mannheim: Universität, Lehrstuhl für Romanistik: 209-230.

LiNK, J. (1988): Literaturanalyse als Interdiskursanalyse am Beispiel des Ursprungs literarischer Symbolik in der Kollektivsymbolik. - In: Fohrmann, J. \& H. MülleR (Hrsg.): Diskurstheorien und Literaturwissenschaft. - Frankfurt am Main: 284-307.

Link, J. \& LinK-HeER, U. (1990): Diskurs/Interdiskurs und Literaturanalyse. - In: Zeitschrift für Literaturwissenschaft und Linguistik 77: 88-99.

LINK, J. \& W. WüLFING (Hrsg.) (1984): Bewegung und Stillstand in Metaphern und Mythen. Fallstudie zum Verhältnis von elementarem Wissen und Literatur im 19. Jahrhundert. - Stuttgart: Klett-Cotta.

Marcus, C.C. (1990): The Garden as Metaphor. - In: Francis, M. \& R.T. Hesters jr. (Hrsg.) (1990): The Meaning of Gardens. - London: 26-33.

PACZIA, D. (1998): Die Natur der Vorgärten. Eine hermeneutisch-geographische Untersuchung eines ästhetischen Phänomens. - Diplomarbeit, Universität Hamburg, Fachbereich Geographie.

PARR, R. (1992): «Zwei Seelen wohnen, ach! in meiner Brust»: Strukturen und Funktionen der Mythisierung Bismarcks (1860-1918). - München: Wilhelm Fink Verlag.

Prest, J. (1981): The Garden of Eden. The Botanic 
Garden and the Re-Creation of Paradise. - New Haven und London: Yale University Press.

Sackville-West, V. (1946): The Garden. - London: Michael Joseph.

SACKVILle-West, V. (1988): Aus meinem Garten. Frankfurt am Main und Berlin: Ullstein.

Scotr-James, A. (1987): Sissinghurst: The Making of a Garden. - London: Michael Joseph.

Strassel, J. (1991): Englische Gärten des 20. Jahrhunderts. - Köln: DuMont.

Strassel, J. (1992): Gärten erleben. - In: Praxis Geographie 5: 16-21.

StRASSEL, J. (1993): Die private Natur des Bürgers. Zur Gartenarchitektur des E. L. Lutyens. - In: JÜNGST, P. \& O. Meder (Hrsg.) (1993): Zur psychosozialen Konstitution des Territoriums. - Kassel: 67-104.

WÜlfing, W., Bruns, K. \& R. PARR (1991): Historische Mythologie der Deutschen, 1798-1918. - München: Wilhelm Fink.

\section{Zusammenfassung: Der Garten als Mythos}

Gärten sind, trotz ihrer wachsenden kulturellen Bedeutung ein wenig beachteter Gegenstand der Geographie. Als zu ihrer Erforschung geeignete theoretische Ansätze werden die strukturalistische Mythentheorie von Claude LÉvi-Strauss und der Diskursbegriff von Michel Foucault vorgestellt und diskutiert. Der Garten von Sissinghurst Castle, England, dient als Beispiel einer zu solcher Analyse relevanten Gartengestaltung.

\section{Summary: The garden as myth}

Despite the increasing cultural significance of gardens, there is little geographic research on the topic. Two approaches suitable for the research of gardens, i.e. Claude LÉvi-STRAuSs' structuralistic theory of myths and Michel Foucault's theory of discourse are outlined and discussed in this paper. The design of the garden in Sissinghurst Castle, England, exemplifies the type of garden these theories are suitable for.

\section{Résumé: Le jardin, un mythe}

Bien que leur importance culturelle augmente, les jardins sont des objets peu considérés par la géographie. La théorie structuraliste des mythes de Claude LÉvIStrauss ainsi que la théorie du discours de Michel Foucault sont présentées et discutées comme bases théoriques. Le jardin de Sissinghurst Castle en Angleterre est un exemple d'aménagement qui se prête bien à une telle analyse.

Prof. Dr. Jürgen Strassel, Carl von Ossietzky Universität Oldenburg, FB 3 - Geographie, Uhlhornsweg, D-20126 Oldenburg.

e-mail: juergen.strassel@uni-oldenburg.de 